\title{
Cramer-Rao lower bound for wideband ToA estimation in the presence of $R F$ nonideality
}

\author{
Tiandong Wang, Yukui Pei ${ }^{a)}$, and Ning Ge \\ Tsinghua National Lab for Info. Science and Technology, Beijing, China \\ a)peiyk@mail.tsinghua.edu.cn
}

\begin{abstract}
In wideband localization systems based on time of arrival (ToA), RF nonideality, such as carrier phase noise, IQ imbalance, phase and frequency offset, plays an important role for ToA estimation accuracy. In this work, Cramer-Rao lower bound (CRB) for ToA estimation in the presence of RF nonideality is first proposed as criterion for ToA estimator performance in practical ToA localization system. Evaluation of CRB with and without phase noise has been performed, especially closed form of CRB without phase noise has been derived. Theoretical and numerical results show that the ToA accuracy presents floor phenomenon in the presence of RF nonideality, and phase noise plays the dominant role. The effect of record length has also been evaluated.
\end{abstract}

Keywords: Cramer-Rao lower bound, RF nonideality, phase noise (PN), time of arrival (ToA), wideband localization

Classification: Navigation, Guidance and Control Systems

\section{References}

[1] H. L. Van Trees, Detection, Estimation, and Modulation Theory, Wiley.com, 2004.

[2] D. Dardari, A. Conti, U. Ferner, A. Giorgetti, and M. Z. Win, "Ranging with ultrawide bandwidth signals in multipath environments," Proc. IEEE, vol. 97, no. 2, pp. 404-426, 2009. DOI:10.1109/JPROC.2008.2008846

[3] Y. Shen and M. Z. Win, "Fundamental limits of wideband localization - part I: a general framework," IEEE Trans. Inf. Theory, vol. 56, no. 10, pp. 4956-4980, 2010. DOI:10.1109/TIT.2010.2060110

[4] A. Conti, M. Guerra, D. Dardari, N. Decarli, and M. Z. Win, "Network experimentation for cooperative localization," IEEE J. Sel. Areas Commun., vol. 30, no. 2, pp. 467-475, 2012. DOI:10.1109/JSAC.2012.120227

[5] P. Steggles and S. Gschwind, "The ubisense smart space platform," Adjunct Proc. of the Third International Conference on Pervasive Computing, pp. 73-76, 2005.

[6] Multispectral Solutions, Inc., "Sapphire dart positioning system."

[7] A. A. D'Amico, L. Taponecco, and U. Mengali, "Ultra-wideband TOA estimation in the presence of clock frequency offset," IEEE Trans. Wireless Commun., vol. 12, no. 4, pp. 1606-1616, 2013. DOI:10.1109/TWC.2013.013013.120319

[8] C. S. Choi, Y. Shoji, H. Harada, R. Funada, S. Kato, K. Maruhashi, I. Toyoda, and K. Takahashi, "RF impairment models for $60 \mathrm{GHz}$-band SYS/PHY simulation," IEEE doc, no.802.15-06, pp. 0477-01-003c, 2006. 
[9] S. M. Kay, Fundamentals of Statistical Signal Processing, Volume 2: Detection Theory, Prentice Hall PTR, 1998.

\section{Introduction}

The Cramer-Rao lower bound (CRB) is a well known lower bound on variance of unbiased estimation on any deterministic parameter, and serves as a benchmark of practical estimators [1]. Time of arrival (ToA) is one of the fundamental parameters of time-of-flight based wideband localization [2]. In previous papers, the CRB of ToA depends on the effective bandwidth of the transmitted signal, the signal to noise ratio (SNR) [2], and path overlap coefficient (in multipath environment) [3]. Based on these three factors, in [2], the root mean square error (RMSE, the square root of $\mathrm{CRB}$ ) of ToA achieves $10^{-10}$ seconds when high SNR (above $13 \mathrm{~dB}$ ), corresponding to less than $3 \mathrm{~cm}$ ranging error. ${ }^{1}$ Moreover, when SNR goes higher, the ranging error goes to less than $1 \mathrm{~cm}$.

However, in real scenarios [4, 5, 6], error of ranging (or localization) is often tens of centimeters, ten times above the CRB proposed by [2]. For in real scenarios, apart from multipath, there are a lot of non-ideal hardware factors that affect the practical system performance, such as clock drift and jitter, carrier frequency and phase offset, phase noise (PN) and IQ imbalance. If these factors are ignored, the CRB of ToA may not be functional to the practical systems. In [7], the effect of clock frequency offsets for ultra-wideband TOA estimation was investigated, and a novel algorithm was proposed to estimate and compensate the clock difference. For other factors, there are few literatures. Moreover, in real scenarios, the record length is finite, while the bandlimited signal is infinite long. Thus the record length greatly affects the performance of ToA localization systems. Meanwhile, many simplified ToA estimation methods [2] use finite samples to estimate ToA. Since long record length leads to the increase of calculation burden, it is of great significance to investigate the effect of record length on ToA estimation.

In this paper, we investigate an analytical expression of the CRB on ToA with carrier frequency and phase offset, IQ imbalance in AWGN channel, followed by CRB analysis with carrier phase noise in AWGN channel.

This paper is organized as follows: in Section II, the ToA system model will be introduced; analysis on CRB of ToA will be presented in Section III; in Section IV numerical results will be presented; and the conclusion goes to Section V.

Notations: $\mathbb{E}_{\mathbf{z}}\{\cdot\}$ is the notation of the expectation operator with respect to random vector $\mathbf{z} ;(\cdot)^{H}$ is complex conjugate transpose of its argument; $\operatorname{tr}\{\cdot\}$ is the trace of a square matrix; $\operatorname{Re}\{\cdot\}$ is the real part of its argument; $[\cdot]_{i, j}$ is the element at the $i$ th row and $j$ th column of its argument; $\mathbb{Z}$ denotes the set of integer.

\section{System model}

We consider a scenario where a pulse ${ }^{2} s(t)$ of which the energy is denoted as $E$ is first passed to an non-ideal radio front-end (RF) through both in-phase and

${ }^{1}$ in the condition of UWB CM4 channel model and with 1 ns pulse width.

${ }^{2}$ In general, $s(t)$ can be a sequence modulated by shaping pulse, e.g. root raised cosine pulse. For bandlimited signal, we consider the sampling interval contains most energy of the signal. 
quadrature channel, then transmitted through a channel with additive white Gaussian noise (AWGN). At last, it is received by another non-ideal RF and sampled with sampling period $T_{s}$. At receiver front-end, the arriving signal is

$$
x(t)=\operatorname{Re}\left\{(s(t)+j s(t)) c_{u}(t)\right\},
$$

where $c_{u}(t)$ is the up-conversion carrier expressed as

$$
\begin{aligned}
c_{u}(t)= & E_{u}\left(\left(1+\frac{\alpha_{u}}{2}\right) \cos \left(\omega t+\phi_{u}(t)+\frac{\theta_{u}}{2}\right)\right. \\
& \left.+j\left(1-\frac{\alpha_{u}}{2}\right) \sin \left(\omega t+\phi_{u}(t)-\frac{\theta_{u}}{2}\right)\right) .
\end{aligned}
$$

In (2), $E_{u}$ stands for the carrier amplitude; $\alpha_{u}$ and $\theta_{u}$ stand for IQ amplitude and phase imbalance; $\phi_{u}(t)$ is carrier phase noise and $\omega$ is the carrier angular frequency. For down-conversion carrier, the corresponding parameters are denoted as $c_{d}(t), E_{d}$, $\alpha_{d}, \phi_{d}(t)$, and $\theta_{d}$. Moreover, the frequency and phase offset are denoted as $\omega_{0}$ and $\psi$. That is, the I and Q channel down-converted signal $r_{I}^{\prime}(t)$ and $r_{Q}^{\prime}(t)$ can be acquired by $r_{I}(t)$ and $r_{Q}(t)$ in (3) with cancelling the term of passband signal at $2 \omega$.

$$
\left\{\begin{array}{l}
r_{I}(t)=x(t-\tau) \cos \left(\left(\omega+\omega_{0}\right) t+\phi_{d}(t)+\psi+\frac{\theta_{d}}{2}\right) \\
r_{Q}(t)=x(t-\tau) \sin \left(\left(\omega+\omega_{0}\right) t+\phi_{d}(t)+\psi-\frac{\theta_{d}}{2}\right) .
\end{array}\right.
$$

Since the phase noise is very small, i.e. $\phi(t)$ is concentrated in a very small range with large probability, we use the approximation with first order Taylor series expansion $^{3} \sin (\phi(t)) \approx \phi(t)$ and $\cos (\phi(t)) \approx 1$. When finished down-conversion, after some trigonometrical transformation and some algebra, the received signal becomes

$$
\begin{aligned}
r(t) & =r_{I}^{\prime}(t)+j r_{Q}^{\prime}(t) \\
& =\mu(t)+v(t)\left(\phi_{u}(t)-\phi_{d}(t)\right)+n(t) .
\end{aligned}
$$

where $n(t)$ is the white Gaussian noise of which the variance is $\sigma^{2}$ and the power spectral density is $N_{0}$, and the $\mu(t)$ and $v(t)$ are

$$
\begin{aligned}
\mu(t)= & s(t-\tau) E_{\mu}\left(\left(1+\frac{\gamma_{\mu}}{2}\right) \cos \left(\omega_{0} t+\Psi_{\mu}+\frac{\Phi_{\mu}}{2}\right)\right. \\
& \left.+j\left(1-\frac{\gamma_{\mu}}{2}\right) \sin \left(\omega_{0} t+\Psi_{\mu}-\frac{\Phi_{\mu}}{2}\right)\right) \\
v(t)= & s(t-\tau) E_{v}\left(\left(1+\frac{\gamma_{v}}{2}\right) \cos \left(\omega_{0} t+\Psi_{v}+\frac{\Phi_{v}}{2}\right)\right. \\
& \left.+j\left(1-\frac{\gamma_{v}}{2}\right) \sin \left(\omega_{0} t+\Psi_{v}-\frac{\Phi_{v}}{2}\right)\right) .
\end{aligned}
$$

${ }^{3}$ In the following paragraph, we assume the distribution of $\phi(t)$ is Gaussian. It's true the range of $\phi(t)$ is from $-\infty$ to $+\infty$ while $\sin (\phi(t))$ is from -1 to +1 , which makes this approximation seem unreasonable. However, Since $\phi(t)$ is very small, the probability of large $\phi(t)$ can be neglected in real scenario. 
(5) shows a form of signal multiplied by non-ideal carrier, where the parameters $E_{\mu}, E_{v}, \gamma_{\mu}, \gamma_{v}, \Phi_{\mu}, \Phi_{v}, \Psi_{\mu}$ and $\Psi_{v}$ are the function of $E_{u}, E_{d}, \alpha_{u}, \alpha_{d}, \theta_{u}, \theta_{d}$ and $\psi$. See Appendix A for detailed derivation.

For phase noise, we assume $\phi_{u}(t)$ and $\phi_{d}(t)$ are i.i.d and generated by phase locked loop (PLL) which subject to zero mean Gaussian distribution, and use onepole and one-zero behavioral model [8] of which the power spectrum density function $\operatorname{PSD}(f)$ is shown as (6), where $K$ is the low frequency phase noise, $f_{p}$ denotes the pole frequency determined by the cutoff frequency of the high-pass filter given by the PLL, and $f_{z}$ is the zero frequency determined by the noise floor of the VCO [8].

$$
\operatorname{PSD}(f)=K \frac{1+\left(f / f_{z}\right)^{2}}{1+\left(f / f_{p}\right)^{2}}
$$

After sampled with the sampling period $T_{s}$, the received signal is shown as (7). Rewritten in form of vector, the received signal becomes (8), where $\mathbf{r}=$ $\left[r(0), \ldots, r\left((N-1) T_{s}\right)\right]^{T}, \quad \boldsymbol{\mu}=\left[\mu(0), \ldots, \mu\left((N-1) T_{s}\right)\right]^{T}, \quad \boldsymbol{v}=\operatorname{diag}(v(0), \ldots$, $\left.v\left((N-1) T_{s}\right)\right), \quad \phi=\left[\phi_{u}(0)-\phi_{d}(0), \ldots, \phi_{u}\left((N-1) T_{s}\right)-\phi_{d}\left((N-1) T_{s}\right)\right]$ and $\mathbf{n}=$ $\left[n(0), \ldots, n\left((N-1) T_{s}\right)\right]$. The covariance matrix of $\boldsymbol{\phi}$ is denoted as $\mathbf{R}$.

$$
\begin{gathered}
r\left(m T_{s}\right)=\mu\left(m T_{s}\right)+v\left(m T_{s}\right)\left(\phi_{u}\left(m T_{s}\right)-\phi_{d}\left(m T_{s}\right)\right) \\
+n\left(m T_{s}\right), m=0,1, \ldots, N-1 . \\
\mathbf{r}=\boldsymbol{\mu}+\boldsymbol{v} \boldsymbol{\phi}+\mathbf{n} .
\end{gathered}
$$

Since $\boldsymbol{\phi}$ and $\mathbf{n}$ are subject to Gaussian distribution, $\mathbf{r}$ is still a Gaussian process. In this paper, we assume that the parameters excluding the time delay $\tau$ have been already known. Thus, for Gaussian process $\mathbf{r}$ [9], the CRB of $\tau$ is

$$
C R B(\tau)=\left(\operatorname{tr}\left[\left(\mathbf{C}^{-1} \frac{\partial \mathbf{C}}{\partial \tau}\right)^{2}\right]+2 \operatorname{Re}\left[\frac{\partial \boldsymbol{\mu}^{H}}{\partial \tau} \mathbf{C}^{-1} \frac{\partial \boldsymbol{\mu}}{\partial \tau}\right]\right)^{-1},
$$

where $\mathbf{C}$ is the covariance matrix of $\mathbf{r}$.

\section{Evaluation of CRB}

\subsection{CRB without carrier phase noise}

If the carrier phase noise is small enough, i.e., $K$ is small enough so that the term $v(t)\left(\phi_{u}(t)-\phi_{d}(t)\right)$ can be ignored. Thus the $\mathbf{C}$ in (9) is $\sigma^{2} \mathbf{I}$, and the CRB of $\tau$ is

$$
\begin{aligned}
C R B(\tau) & =\left(2 \operatorname{Re}\left[\frac{\partial \boldsymbol{\mu}^{H}}{\partial \tau} \mathbf{C}^{-1} \frac{\partial \boldsymbol{\mu}}{\partial \tau}\right]\right)^{-1} \\
& =\frac{2 \sigma^{2}}{\left.\sum_{n=0}^{N-1}\left|\frac{\partial r(t-\tau)}{\partial \tau}\right|_{t=n T_{s}}\right|^{2}} .
\end{aligned}
$$

If the sampling period is small enough, i.e., when $T_{s} \rightarrow 0$, using Fourier Transformation property and Parseval's Theorem [9] (Appendix B), the $C R B(\tau)$ becomes

$$
C R B(\tau)=\frac{1}{\beta_{R F} E / N_{0}} .
$$


$\int_{T_{s}} s(t)^{2} d t=\int_{-\infty}^{\infty}|S(\omega)|^{2} d \omega$, and the $\beta_{R F}$ is the effective bandwidth in the condition of non-ideal RF in (12).

$$
\begin{aligned}
& \beta_{R F}= \\
& \frac{\left(\begin{array}{c}
\frac{E_{\mu}^{2}}{\pi}\left(1+\frac{\gamma_{\mu}}{2}\right) \int_{-\infty}^{\infty}\left|\left(\omega-\omega_{0}\right) S\left(\omega-\omega_{0}\right) e^{-j\left(\Psi_{\mu}+\frac{\Phi_{\mu}}{2}\right)}+\left(\omega+\omega_{0}\right) S\left(\omega+\omega_{0}\right) e^{j\left(\Psi_{\mu}+\frac{\Phi_{\mu}}{2}\right)}\right|^{2} d \omega \\
+\frac{E_{\mu}^{2}}{\pi}\left(1-\frac{\gamma_{\mu}}{2}\right) \int_{-\infty}^{\infty}\left|\left(\omega-\omega_{0}\right) S\left(\omega-\omega_{0}\right) e^{-j\left(\Psi_{\mu}-\frac{\Phi_{\mu}}{2}\right)}+\left(\omega+\omega_{0}\right) S\left(\omega+\omega_{0}\right) e^{j\left(\Psi_{\mu}-\frac{\Phi_{\mu}}{2}\right)}\right|^{2} d \omega
\end{array}\right)}{4 \int_{-\infty}^{\infty}|S(\omega)|^{2} d \omega}
\end{aligned}
$$

Remark: Comparing to the effective bandwidth of ideal RF (13), the $\beta_{R F}$ is the weighted average of frequency shifted version of $\omega S(\omega)$. The weighting factors depends on the non-ideal RF parameters.

$$
\beta_{\text {ideal }}=\frac{\int_{-\infty}^{\infty}|\omega S(\omega)|^{2} d \omega}{\int_{-\infty}^{\infty}|S(\omega)|^{2} d \omega}
$$

\subsection{CRB with carrier phase noise}

If $K$ in (6) is large enough, which makes phase noise non-negligible, the covariance matrix $\mathbf{C}$ in (9) will not be $\sigma^{2} \mathbf{I}$ any more. Moreover, $\mathbf{C}$ will depend on $\tau$ due to $s(t-\tau)$ in term $\boldsymbol{v}$ in (8). And the combined noise $\boldsymbol{v} \boldsymbol{\phi}+\mathbf{n}$ becomes colored noise. In this condition, the CRB can hardly be expressed in a closed form. Since $\boldsymbol{\phi}$ and $\mathbf{n}$ are independent, $\mathbf{C}$ becomes

$$
\begin{aligned}
\mathbf{C} & =\mathbb{E}_{\boldsymbol{\phi}, \mathbf{n}}\left\{(\boldsymbol{v} \boldsymbol{\phi}+\mathbf{n})(\boldsymbol{v} \boldsymbol{\phi}+\mathbf{n})^{H}\right\} \\
& =\boldsymbol{v} \mathbf{R} \boldsymbol{v}^{H}+\sigma^{2} \mathbf{I} .
\end{aligned}
$$

According to the one-zero and one-pole carrier phase noise model (6), the auto correlation function of $\boldsymbol{\phi}$ will be (15), where $\delta(\cdot)$ is the delta function and $m \in \mathbb{Z}$. Thus $[\mathbf{R}]_{i, j}=R(i-j)$.

$$
R(m)=K\left[\frac{f_{p}^{2}}{f_{z}^{2}} \delta(m)+\pi\left(\frac{f_{p}^{2} f_{z}^{2}-f_{p}^{4}}{f_{p} f_{z}^{2}}\right) e^{-2 \pi f_{p} T_{s}|m|}\right] .
$$

Therefore, the CRB of $\tau$ can be calculated using (9), (14) and (15).

\section{Numerical results}

We use root raised cosine (RRC) pulse as $s(t)$ to calculate the CRB.

Fig. 1 shows the relationship between SNR and RMSE in different scenarios. In this result, The RRC rolloff factor is 0.5 ; the symbol rate is $300 \mathrm{MHz}$; the sampling rate is $900 \mathrm{MHz}$; and the waveform record length is 2 symbols. The phase noise factor $K$ is $-40 \mathrm{~dB}$; and the $f_{p}$ is $10 \mathrm{kHz} ; f_{z}$ is $1 \mathrm{MHz}$. This result shows that by adding non-ideal RF without PN, the RMSE will raise up due to the change from $\beta_{\text {ideal }}$ to $\beta_{R F}$. Moreover, when added PN, the RMSE will raise more in high SNR region, i.e., the system performance flattens. This is because in high SNR region, the effect of white Gaussian noise is much less than that of PN, and in (15), there is a term of $\delta(m)$, which is stands for white noise part of phase noise. Therefore, although white Gaussian noise is decreasing, phase noise limits the 


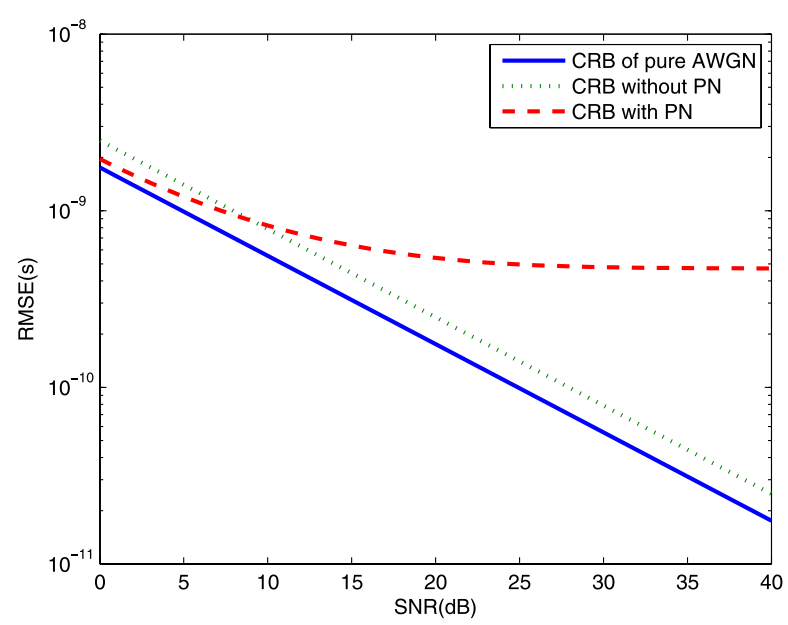

Fig. 1. CRB of ToA in 3 different scenarios: $C R B$ in the assumption of pure AWGN channel, CRB without phase noise, and CRB with affection of phase noise.

performance of the ToA system. Moreover, the exponential term in (15) is also a limitation of the performance.

In low SNR region RMSE with PN is smaller than that without PN. This is because in (14), $v$ carries the information of $\tau$, which makes RMSE with PN a little smaller than that without PN. However, it is well-known that in low SNR region, CRB is not a tight bound [2], so it is not of great significance to pay attention on CRB of low SNR region.

Fig. 2 reveals in the condition of different $K$, the relationship between SNR and RMSE. When $K$ decreases, the floor of CRB decreases. Thus the strength of phase noise $K$ becomes a bottom neck that affects the performance of ToA localization system in high SNR region. The values of other parameters are same as Fig. 1.

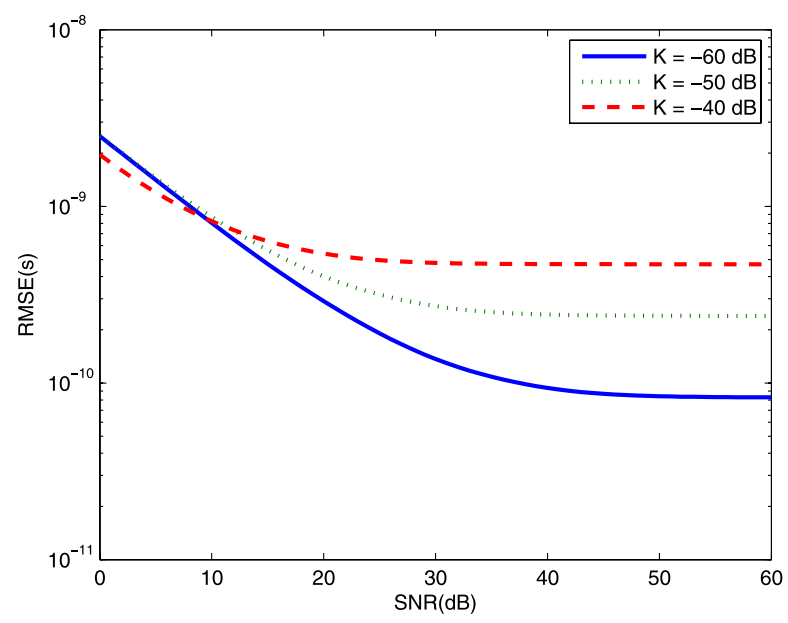

Fig. 2. $\mathrm{CRB}$ of ToA with different phase noise level by adjusting $\mathrm{K}$ level. The floor of CRB drops off as K decreases.

Fig. 3 shows that long record length can increase the performance of ToA estimation. However, it is shown that increase from short record length ( 2 symbols) to middle one (4 symbols) leads to great performance improvement, while that from 
middle one to long one ( 8 symbols) leads to little gain. This is because most (e.g. $95 \%$ ) energy of the pulse is concentred on a finite time interval. When the record length increases, the energy of the record signal does not increase so rapidly as the record length does.

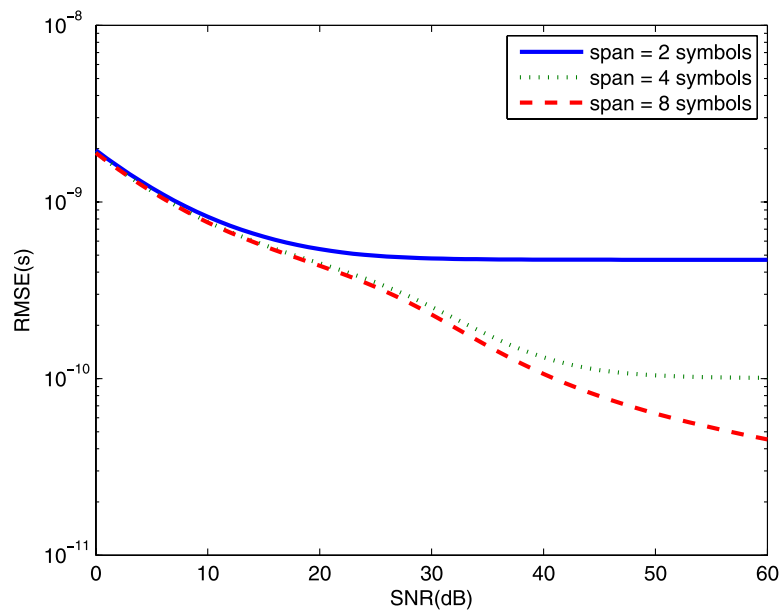

Fig. 3. CRB of ToA with different record length.

These numerical results provide a reason why in real system, the error of ranging or localization system is often at the level of decimeter $\left(10^{-9}\right.$ seconds correspond to $30 \mathrm{~cm}$ ). There certainly exist other factors that limit the performance, such as clock drift, ADC converter error and so on, but non-ideal RF and record length are significant factors.

\section{Conclusion}

In wideband ToA localization system, RF nonideality greatly affects ToA estimation performance. Evaluation of $\mathrm{CRB}$ on ToA estimation in the presence of RF nonideality provides criterion to ToA localization design and ToA estimator performance, which gives a reasonable way to improve the ranging accuracy for real localization systems.

\section{A Received signal calculation}

After expanding (3), cancelling $2 \omega$ term, and approximating using Taylor series expansion, $r(t)$ can be seperated in three parts which are $\mu(t), v(t)\left(\phi_{u}(t)-\phi_{d}(t)\right)$, and $n(t) . \operatorname{Re}\{\mu(t)\}$ is shown as (16) and (17).

$$
\begin{aligned}
\operatorname{Re}\{\mu(t)\}= & {\left[\frac{(\beta+2)(\alpha+2)}{8} \cos \left(\omega_{0} t+\psi_{0}-\frac{\theta_{1}-\theta_{2}}{2}\right)\right.} \\
& \left.+\frac{(\beta-2)(\alpha+2)}{8} \sin \left(\omega_{0} t+\psi_{0}+\frac{\theta_{1}+\theta_{2}}{2}\right)\right] \\
& \cdot E_{u} E_{d} s(t-\tau) .
\end{aligned}
$$

After reformulating $\operatorname{Re}\{\mu(t)\}$ and $\operatorname{Im}\{\mu(t)\}$ into form of $A \cos (\mathrm{a})$ and $B \cos (\mathrm{b})$, respectively, we have form of (5), which is the same form of IQ imbalance, 
frequency and phase offset carrier form. And the $v(t)$ is derived as the same way as $\mu(t)$.

$$
\begin{aligned}
\operatorname{Im}\{\mu(t)\}= & {\left[\frac{(\beta-2)(\alpha-2)}{8} \cos \left(\omega_{0} t+\psi_{0}+\frac{\theta_{1}-\theta_{2}}{2}\right)\right.} \\
& \left.+\frac{(\beta-2)(\alpha+2)}{8} \sin \left(\omega_{0} t+\psi_{0}-\frac{\theta_{1}+\theta_{2}}{2}\right)\right] \\
& \cdot E_{u} E_{d} S(t-\tau) .
\end{aligned}
$$

\section{B CRB of continuous-time without phase noise}

According to (10):

$$
\begin{aligned}
\lim _{T_{s} \rightarrow 0} C R B(\tau) & =\lim _{T_{s} \rightarrow 0} \frac{2 \sigma^{2}}{\left.\sum_{n=0}^{N-1}\left|\frac{\partial r(t-\tau)}{\partial \tau}\right|_{t=n T_{s}}\right|^{2}} \\
& =\lim _{T_{s} \rightarrow 0} \frac{2 \sigma^{2} T_{s}}{\left.\sum_{n=0}^{N-1} T_{S}\left|\frac{\partial r(t-\tau)}{\partial \tau}\right|_{t=n T_{s}}\right|^{2}} \\
& =\frac{2 N_{0}}{\int_{T_{r}}\left|\frac{\partial r(t-\tau)}{\partial \tau}\right|^{2} d t} .
\end{aligned}
$$

$T_{r}$ stands for record length. Using the Parseval's Theorem and relation $\int\left|\frac{\partial s(t-\tau)}{\partial \tau}\right|^{2} d t=\int\left|s^{\prime}(t)\right|^{2} d t$, we have:

$$
\begin{aligned}
\int_{T_{r}}\left|\frac{\partial r(t-\tau)}{\partial \tau}\right|^{2} d t & =\int_{T_{r}}\left|s^{\prime}(t) c_{\mu}(t)\right|^{2} d t \\
& =\int_{-\infty}^{\infty}\left|(\omega S(\omega)) * C_{\mu}(\omega)\right|^{2} d \omega
\end{aligned}
$$

In (19) $c_{\mu}(t)=\mu(t) / s(t-\tau)$, and $*$ stands for convolution. Thus we have (12).

\section{Acknowledgments}

This research is supported by the National Science and Technology Major Project No. 2011ZX03004-001-01, National Nature Science Foundation of China No. 61128001, No. 61132002, No. 60928001 and No. 60972019. 